\title{
Improved Digital Image Compression using Modified Single Layer Linear Neural Networks
}

\author{
B.Arunapriya \\ Research Scholar \\ Bharathiyar University, PSGR \\ Krishnammal College for Women, \\ Coimbatore, Tamilnadu, India.
}

\author{
D.KavithaDevi \\ Assistant Professor \\ Bharathiyar University, PSGR \\ Krishnammal College for Women, \\ Coimbatore, Tamilnadu, India.
}

\begin{abstract}
Image Compression is solved by using Wavelet-Modified Single Layer Linear Forward Only Counter Propagation Network (MSLLFOCPN) technique. Form the wavelets it inherits the properties of localizing the global spatial and frequency correlation from wavelets. Function approximation and prediction are obtained from neural networks. As a result counter propagation network was considered for its superior performance and the research enable us to propose a new neural network architecture named single layer linear counter propagation network (SLLC). The combination of wavelet and SLLC network were tested on several benchmark images and the experimental results shows that an enhancement in picture quality, compression ratio and approximation or prediction comparable to existing and traditional neural networks.
\end{abstract}

\section{Keywords}

Wavelet, Modified Single Layer Linear Forward Only Counter propagation; Clustering; Distance Metrics.

\section{INTRODUCTION}

Modern application requires high volume of data to accommodate large number of images. Image compression represents the process of data reduction and at the same time retains image information. The key components to compress the data are specified channel bandwidths or storage requirements and maintaining the highest possible quality. So efficient data compression techniques save storage space and accelerate the transmission time.

Compression of digital images has been the focus of much recent research interest in today's rapidly changing world. It has basically two inherent benefits. First, it makes possible to use digital images in transmission and storage environment that would not support uncompressed raw images. For example, current Internet throughput rates are insufficient to handle uncompressed image in real time (even at small image size). Second, compression enables more efficient use of transmission and storage resources. If a high bit rate transmission channel is available, then it is more attractive proposition to send high resolution compressed image or multiple compressed images than to send a single, low resolution, uncompressed image. The requirement of enormous expenditure on bandwidth and/or storage necessitates the use of various compression schemes even with constant advances in storage and transmission capacity.

Digital Images contain huge amount of data. So to decrease the memory needed to store the image and to reduce the bandwidth44 requirement to transmit, it is essential to compress, it as efficiently. Image compression is the representation of an image in digital form with as few bits as possible while maintain an acceptable level of image quality. Many compression techniques have been already proposed in literature by several researchers, such as transform image coding, predicative image coding and vector quantization. Among these, simple and efficient way of performing image compression is transform image coding, particularly at low bit rates. For past several years JPEG (DCT based) image compression has been a standard choice. Wavelet transforms have become the most prevalent techniques among these transform image coding techniques, as they are localized in both image space and spectral frequency domains.

Artificial neural networks are popular and found its application in many fields, mainly in function approximation, due to their ability to approximate complicated nonlinear functions [9]. There are mainly three types of learning used in different application i.e., supervised, unsupervised and reinforcement learning. The multilayer perceptron (MLP) along with the backpropagation (BP) learning algorithm can be categorized as a supervised neural network and most frequently used neural network in practical situations.

Counterpropagation neural network (CPN), a combination of supervised and unsupervised neural net, typically converges much more quickly than multilayer perceptron neural network (MLP NN). Hence, it can be used as an alternative to the MLP trained by BP. Many researchers have used correlation based techniques for training to forward-only counterpropagation network (FOCPN). In Modified FOCPN (MFOCPN) using different distance metrics for selection of winner among the hidden layer neurons and nonlinear learning rates for better performance of the network is proposed. Some recent papers show that the combination of neural network based approach and classical wavelet based approach leads to better compression ratio. A hybrid network is proposed which generates the codebook for wavelet coefficients, obtained from wavelet decomposition of an image. The author have not exploited the spatial and statistical properties of wavelet 
coefficients in wavelet domain across various levels of decomposition and the work was mainly motivated to find the interface between wavelet domain and competitive learning using neural network, like counterpropagation neural network.

Till date, numerous image compression techniques discovered are transform image coding, predicative image coding and vector quantization. Out of these, transform image coding is an efficient technique particularly at low bit rates. JPEG and DCT based image compression has been a standard of choice for decades. However, wavelet transform becomes the most prevalent technique, since they are localized in both spatial and frequency domain [1]. Artificial neural network architectures are used to study the performance in function approximation and prediction due to their ability to approximate complicated function [8]. From the experimental tests, it was found that counter propagation neural network produce more accurate and converges much more quickly than other networks $[9,1]$. The correlation based techniques were used by many researchers for clustering which is limited to forward only counter propagation network. This has its own limitations, the techniques to overcome these limitations are discussed in $[10,1]$. As a result more attention was focused on modification which enhances the performance of counter propagation network. Some recent papers shows that the combination of neural network and classical wavelet based approach leads to better compression ratio [7]. In this work we have integrated wavelet transform to MSLLFOCPN based image compression. Results obtained with proposed technique leads to better compression ratio at the same time preserving the image quality.

This paper is organized as. Section III precisely deals about Wavelet and MSLLFOCPN. Section IV describes proposed methodology and its architecture. Section V tells about the simulation results and Section VI views our conclusion.

\section{RELATED WORK}

Grgic el al[7] .,describes about image compression using discrete wavelet transform (DWT) and represents images as a sum of wavelet functions (wavelets) on different resolution levels. The basis for the wavelet transform can be composed of any function that satisfies requirements of multiresolution analysis. It means that there exists a large selection of wavelet families depending on the choice of wavelet function. The choice of wavelet family depends on the application. In image compression application this choice depends on image content. The author provides fundamentals of wavelet based image compression. The options for wavelet image representations are tested.

Woods et al,.[4] describes about the variations on BPN (backward propagation network) and CPN (counter propagation networks) have been presented. The BPN variations are a little quicker and the CPN variations are a little more accurate. The author suggests that these variations should be tested on some other more difficult problems. To this end, a fault-tolerant (in software) handwriting recognizer is being developed using these BPN and CPN variations, along with an adaptive resonance network. Preliminary results indicate that by using the author's equation with the BPN, a $20 \%$ decrease in learning time can be obtained, which represents a reduction from 30 to 24 hours for learning the alphabet.
Poggio el al,. presents a new learning algorithm for radial basis functions (RBF) neural network, based on robust statistics. The extention of the learning vector quantizer for second order statistics is one of the classical approaches in estimating the parameters of a RBF model. The author provides a comparative study for these two algorithms regarding their application in probability density function estimation. The theoretical bias in estimating one-dimensional Gaussian functions are derived.

Christipher el al,.describes characteristics of providing spatial and frequency information in the transform domain by wavelet transform, plays a crucial role in forming a significance map of coefficients that needs to be coded in the case of image compression. This paper presents a wavelet based image compression technique where transmission of spatial locations of wavelet decomposed lower subbands coefficients is eliminated. The spatial position information of significant coefficients of subbands of each level can be found by interpolation of higher level subbands to lower level at decoder side. This in turn, saves the data corresponding to positional information of significant coefficients, further giving an opportunity to enhance image quality at same compression level by including additional wavelet coefficients. The simulation results show that the combination of interpolation and vector quantization (VQ) using neural networks performs better than baseline JPEG.

Deepak et al,. proposes a modified forward-only counter propagation neural network (MFO-CPN) for color image compression is proposed. It uses several higher-order distance measures for calculating winning node. It also incorporates nonlinear adjustment of learning rates in both the layers. Results with these distance functions are compared. Proposed modifications leads to improvement in the image quality and faster convergence of network.

\section{WAVELET TRANSFORMS AND MSLLFOCPN}

\subsection{Wavelet transforms}

Wavelet transform (WT) of an image represents image as a sum of wavelets on multi-resolution levels. Multi-resolution analysis is implemented via high-pass filters (wavelets) and low-pass filters (scaling functions).

In wavelet transform any one-dimensional function is transformed into a two-dimensional space, where it is approximated by coefficients that depend on time (determined by the translation parameter) and on scale, (determined by the dilation parameter). The zoom phenomena of the WT offer higher temporal localization for high frequencies while offering good frequency resolution for low frequencies. Hence, the wavelet transform is well suited to image compression.

\subsection{MSLLFOCPN}

The original forward-only counter propagation network shown in fig. 1 has only one output unit per each output element. So the cluster has only one average value that represents the average of the correct output value $y$ associated with the input vector value $\mathrm{x}$ that cause the cluster to win the competition. 
This will limit the net capabilities to produce specific output values instead of approximating a function that interacts with the input vectors to determine the output values. The objective of the net is to approximate a continuous function that maps a subset of n-dimensional Euclidean space into the real numbers. Vector functions can be handled by creating one network per output coordinate.

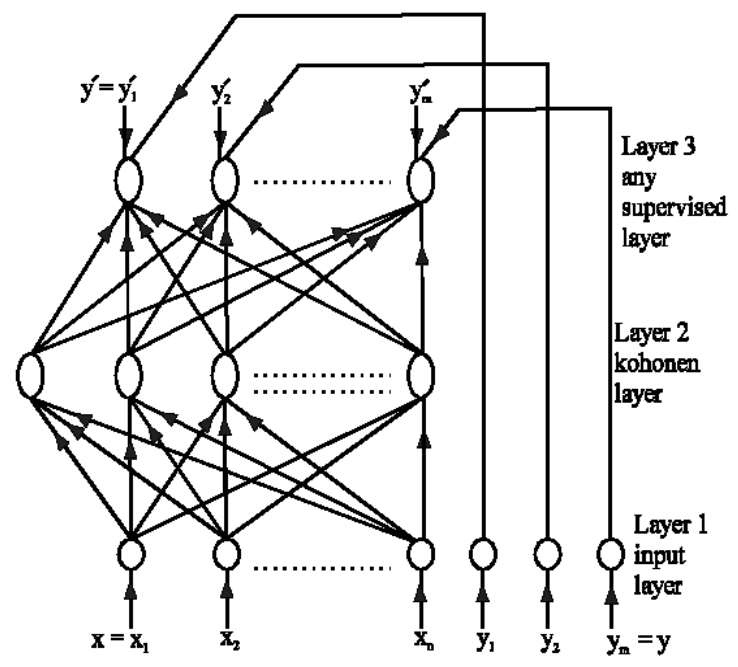

Fig. 1 The Forword Only Counter propagation network

The modification to the original counter propagation network was accomplished by constructing a network for each cluster. Each cluster's network will approximate a supervised learning. As first step, the constructed network will be multi-layered network with two hidden layers network with two hidden layers since cybenko showed that two hidden layers network with continuous sigmoid non linearity can approximate any continuous function arbitrarily well on a compact set. The resulting modified counter propagation network is shown in fig.2. Figure 3 shows the structure of the two hidden layered networks referred to in fig 2.

As a further refinement, the two hidden layers of the network of Fig 3 is replaced by single layer linear network. Although the former is more robust than the later the single layer linear net is simpler, require less processing elements, does not need a normalization process and it is easily understood. In single layer linear network robustness can be achieved by increasing number of clusters in the network.

The final resulting architecture of the single layer linear counter propagation network show in fig 4 consist of a number of cluster's each of these clusters contains single processing elements that approximate a function for the subset of examples associated with that cluster.

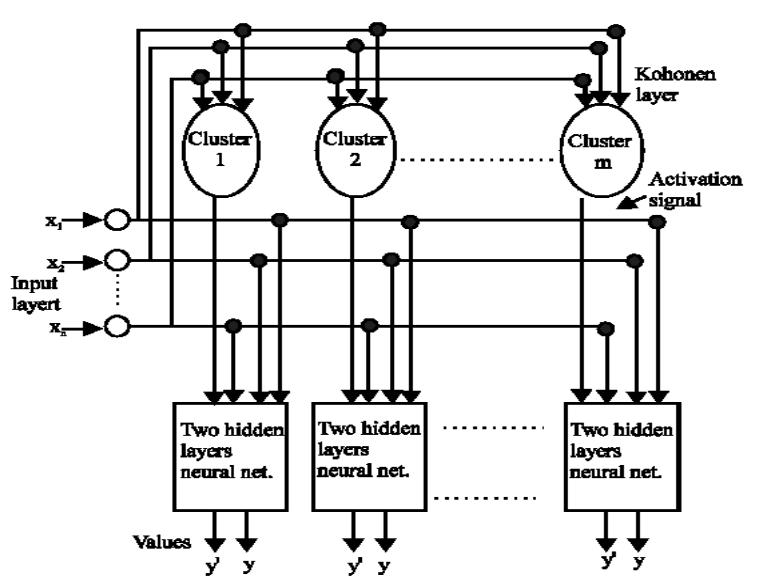

Fig 2: Modified Single Layer Liner Counterpropagating network

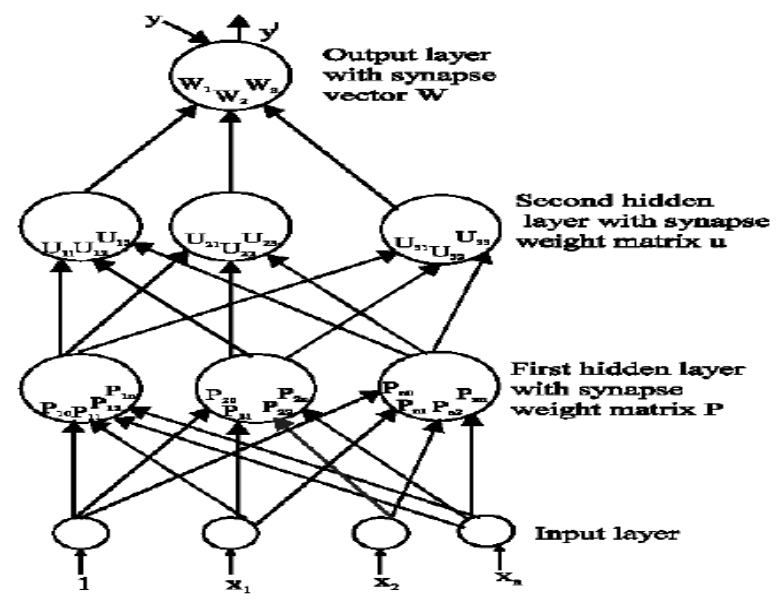

Fig 3: Structure of Two hidden Layer Network

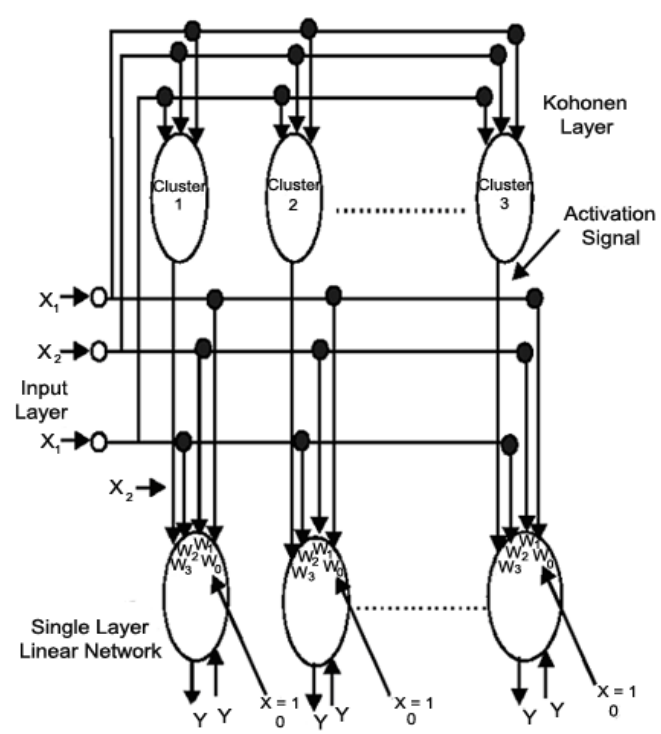

Fig.4: The final structure of SLLIC Network 


\section{PROPOSED METHODOLOGY}

In this paper the use of MSLLFOCPN networks is explored to predict wavelet coefficients for image compression. In this method, we integrate the classical wavelet based method with MSLLFOCPN. Instead of passing whole pixel values of image we pass the significant wavelet coefficients obtained after applying wavelet transform to image. This provides better compression because at one stage compression is achieved by wavelet transform and in next stage compression is done by MSLLFOCPN. Both figures are shown in fig $5 \& 6$.

Wavelet based compression systems are shown in figure 5 . The wavelet coefficients are quantized and are encoded without loss by entropy encoder box, which usually has contextual information. Higher amounts of compression are obtained by increasing the quantization step sizes and by making better prediction for the ranges of quantized values via appropriate contexts and data structures.

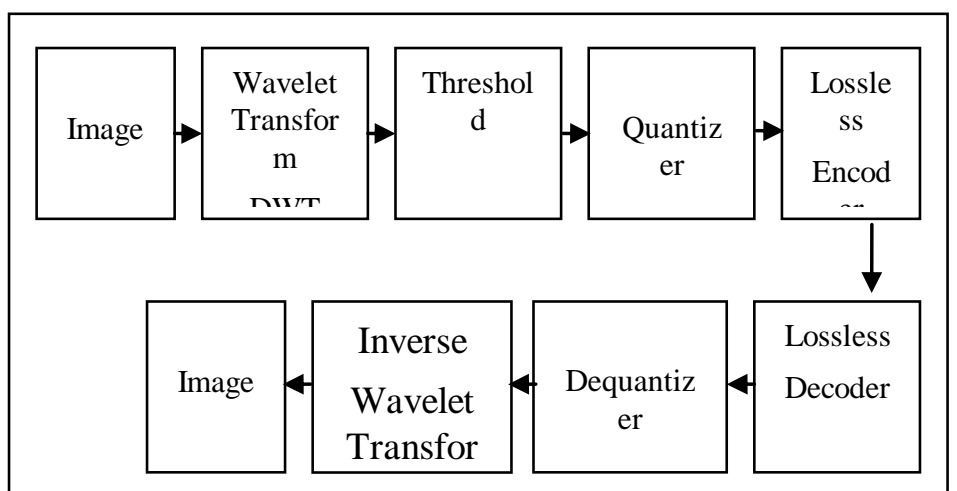

Fig. 5 Block Diagram for Wavelet Based Image Compression

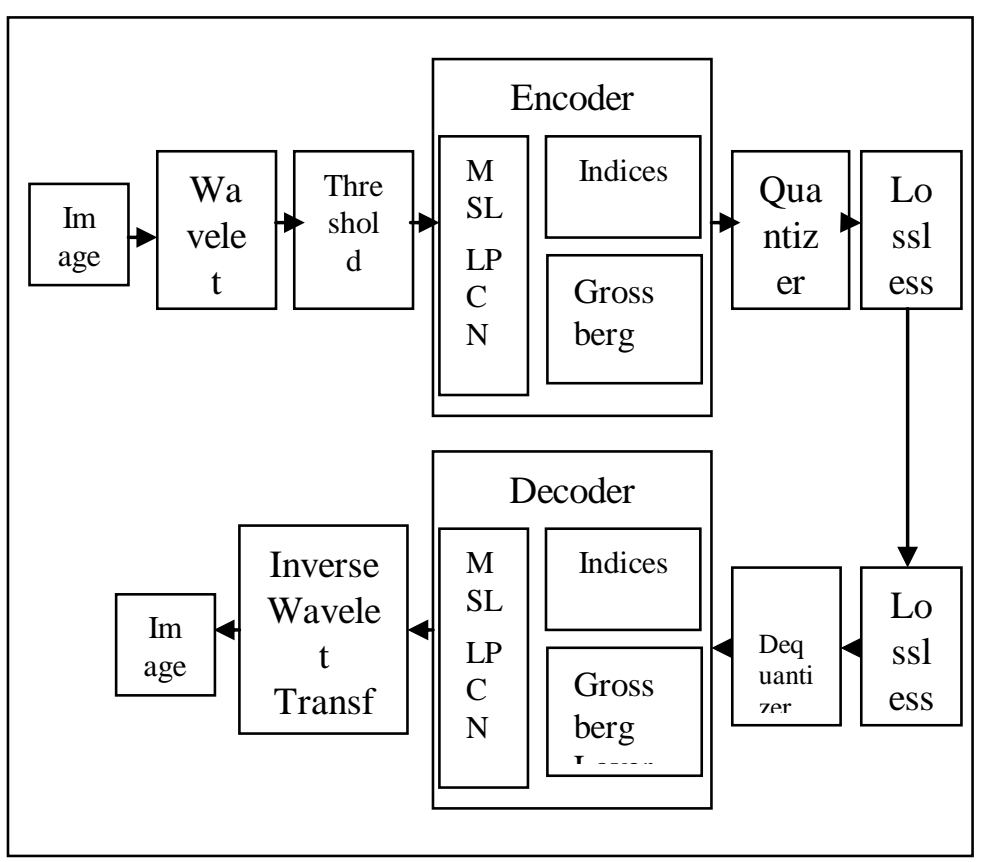

Fig. 6 Block Diagram for Wavelet - CPN Based Image Compression
To achieve better compression MSLLFOCPN is used after wavelet transform block shown in figure 6. The MSLLFOCPN is used for predicting wavelet coefficients and training is done for each wavelet level and subband which is obtained after applying the threshold. Applying threshold is beneficial, that lower sub bands of wavelet has significant information and we only need to learn these important information.

Figure 7 shows typical wavelet subband decomposition. The notation $\mathrm{L}$ and $\mathrm{H}$ stand respectively for lowpass and highpass filtering. The LL wavelet themselves act as smooth predictors and $\mathrm{LH}, \mathrm{HL}$ and $\mathrm{HH}$ coefficients are the residuals computed from these predictors. The MSLLFOCPN basically works as the function approximation and predicts the useful coefficients.

It requires less number of clusters to hold these values and we can store all the useful wavelet coefficients in these cluster indices and grossberg layer weights. Instead of sending whole wavelet coefficients we now send the cluster indices and grossberg layer weights which require less number of bits and we can achieve higher compression ratio. The algorithm used for training of network and Euclidean distance function to find out the winner is presented in figure 8 .

\begin{tabular}{|c|c|c|c|}
\hline LL2 & LH2 & & \\
HL2 & HH & LH1 & \multirow{2}{*}{ LH0 } \\
\cline { 1 - 2 } HL1 & HH1 & \\
\hline \multicolumn{2}{|c|}{ HLO } & \multirow{2}{*}{ HHO } \\
\hline
\end{tabular}

Fig. 7. Multi-resolution wavelet representation (three levels)

Algorithm for implementing Modified Single Layer Linear Counter Propagation Network.

Phase One \{ Kohonen Layer\}

Step 0: Initialize the clusters weight vectors and the learning rate.

Step 1: Repeat from step 2-7 until the weight vectors converge.

Step 2: for each training input vector do steps 3-5.

Step 3: Set the input layer activation's to vector $\mathrm{x}$.

Step 4: Find winning cluster unit using distance measure; call its index $\mathrm{j}$.

Step 5: Update weight vector $\mathrm{v}_{\mathrm{j}}$ of unit $\mathrm{j}$ using:

$\mathrm{V}_{\mathrm{ij}}($ new $)=\mathrm{V}_{\mathrm{ij}}($ old $)+\mathrm{a}\left(\mathrm{x}_{\mathrm{i}} \cdot \mathrm{V}_{\mathrm{ij}}\right.$ (old) $\left.1=1,2, . ., \mathrm{n}\right)$

Step 6: Reduce learning rate a.

Phase Two \{ Single Layer Linear Network\}

Step 0: For each single layer linear net of a cluster do.

Step 1: Intialize weight vector w(randomly) and the learning rate a.

Step 2: set $E=0$ and $K=0$ ( $k$ is a counter for the number of associative examples to the current clusters net)

Step 3: $\mathrm{k}=\mathrm{k}+1$ 


$$
\begin{aligned}
& X=x_{k} \\
& y=y_{k} \\
& y^{\prime}=\sum_{m=0}^{n} W_{m} X_{m} \\
& e=y-y^{\prime} \\
& W j=W j+a^{*|z|^{2}} \\
& y^{\prime}=\sum_{m=0}^{n} W_{M} X_{M} \\
& E=E+a b c\left(y-y^{\prime}\right)
\end{aligned}
$$

Step 4: if $\mathrm{k}<\mathrm{m}$ then go to 3

Step 5: if $\mathrm{E}>\mathrm{E}_{0}$ then go to 2

where

$\mathrm{E}:$ the absolute error of current cluster

$\mathrm{E}_{0}$ : the previous absolute error of the current cluster.

$\mathrm{W}_{\mathrm{j}}$ :the jth weight of the processing element.

$y$ ': the actual output of the cluster.

$\mathrm{y}$ : the desired output

$\mathrm{X}$ : the input vector.

a: the learning rate

M: the number of associated examples with the current cluster net.

\section{SIMULATION RESULTS AND COMPARISON}

In this section, simulation results for the proposed technique with three clusters (namely 32, 64 and 128) of different image size for lena $(256 \times 256,512 \times 512)$ are shown. Quality measures such as PSNR and MSE for decompressed image are calculated and compared. Table $1 \& 2$, shows the comparison of the results with the proposed technique to the existing modified forward only counter propagation network.

Table 1: Performance and comparison of existing and proposed technique for image size $256 \times 256$

\begin{tabular}{|c|c|c|c|}
\hline \multirow{2}{*}{$\begin{array}{c}\text { Image Size } \\
256\end{array}$} & \multicolumn{3}{|c|}{ Wavelet - MFOCPN } \\
\cline { 2 - 4 } & $\begin{array}{c}\text { Cluster } \\
32\end{array}$ & $\begin{array}{c}\text { Cluster } \\
64\end{array}$ & $\begin{array}{c}\text { Cluster } \\
128\end{array}$ \\
\cline { 1 - 4 } Lena & & 24.3541 & 24.4252 \\
\hline PSNR & 24.2335 & 234.3801 & 234.7253 \\
\hline MSE & 234.2800 & 250 \\
\hline
\end{tabular}

Table 2: Performance and comparison of existing and proposed technique for image size $256 \times 256$

\begin{tabular}{|c|c|c|c|}
\hline \multirow{2}{*}{$\begin{array}{c}\text { Image Size } \\
256\end{array}$} & \multicolumn{3}{|c|}{ Wavelet - MSLLFOCPN } \\
\cline { 2 - 4 } Cameraman & Cluster 32 & $\begin{array}{c}\text { Cluster } \\
64\end{array}$ & $\begin{array}{c}\text { Cluster } \\
128\end{array}$ \\
\hline PSNR & 27.9068 & 28.2028 & 28.2233 \\
\hline MSE & 105.294 & 98.356 & 97.8917 \\
\hline
\end{tabular}

\section{CONCLUSION AND DISCUSSION}

In this paper, the proposed technique is used for image compression. The algorithm is tested on varieties of benchmark images. Simulation results for one of the standard image, i.e., Lena with different clusters are presented. These results are compared with existing technique. Several performance measures are used to test the reconstructed image quality.

a) Wavelet-MSLLCPN 256 x 256 with 128 cluster

b) Wavelet-MFOCPN $256 \times 256$ with 128 cluster

c) Wavelet-MFOCPN $512 \times 512$ with 128 cluster

d) Wavelet-MSLLCPN 512 x512 with 128cluster a
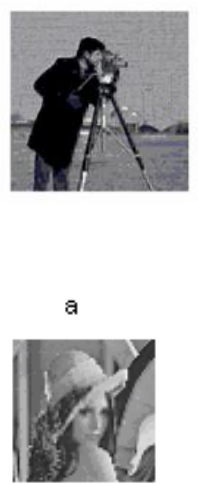

b

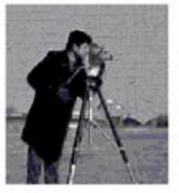

b

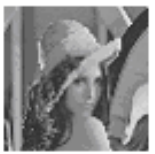

c

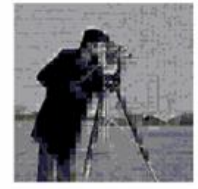

c

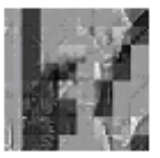

d

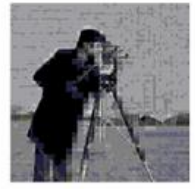

d

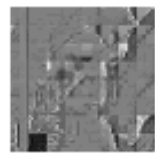

According to the experimental results, the proposed technique outperformed the existing wavelet based image compression. It can be inferred from experimental results as shown in Table 2 that the higher order distance particularly performed well and results higher compression ratio. Besides higher compression ratio it also preserves the quality of the image, as it considers the standard deviation of the inputs to the network and clusters them with more accuracy.

It can be concluded that the integration of classical with soft computing based image compression methods enables a new way for achieving higher compression ratio.

\section{REFERENCES}

[1]A. K. M. Ashikur Rahman and Chowdhury Mofizur Rahman, "A New Approach for Compressing Color Images using Neural Network', CIMCA 2003 Proceedings/ISBN 1740880684: M. Mohammadian (Ed.) 12-14 February 2003, Vienna - Austria

[2]Christopher J.C. Burges, Henrique S. Malvar, Patrice Y. Simard, "Improving Wavelet Image Compression with Neural Networks", MSR-TR-2001-47, August 2001, pp. 118.

[3]D. Salomon, "Data Compression: The Complete Reference", Springer (India) pvt. Ltd., 2005

[4]Qiu, G.; T.J. Terrell; M.R.Varley; "Improved image compression using backpropagation network", workshop on neural network applications and tools, September 13-14, 1993 pp. $73-81$.

[5] Sameh Ghwanmeh; Riyad Al-Shalabi; G.Kana'n and Luai Alnemi;“"Enhanced neural network model based on a single 
layer linear counterpropagation for prediction and function approximation", Information Technology Journal 5(1): 45$50,2006$.

[6]Donald woods, "Back and Counter Propagation Abberations", Proc.IEEE, Neural Networks, Vol. 1 1988, pp. 473-479.

[7]Sonja Grgic, Kresimir Kers, Mislav Grgic. "Image compression using wavelets”, ISIE'99 - Bled, Slovenia, pp. 99-104.

[8]Poggio T., F. Girosi "Networks for approximation and learning”, Proc. IEEE, Vol. 78, no.9, Sept 1990; pp. $1481-1497$
[9]Hornik K," Multilayer feedforward networks are universal approximators", Neural Networks, Vo1.2, no.5, pp. 359366, 1989

[10]Deepak Mishra, N. S. C Bose, A. Tolambiya, A. Dwivedi, P. Kandula, A. Kumar, Prem K. Kalra, "Color Image Compression with Modified Forward-Only Counterpropagation Neural Network: Improvement of the Quality using Different Distance Measures", Proceedings of the 9th IEEE International Conference on Information Technology, ICIT'06, India, (In press). 